\title{
INFLUÊNCIA DO CÁLCULO DE DEPRECIAÇÃO NO IMPOSTO DE RENDA E NO FLUXO DE CAIXA DE UMA ATIVIDADE DE TRANSPORTE FLORESTAL ${ }^{1}$
}

\author{
Luis Carlos de Freitas², Márcio Lopes da Silva ${ }^{3}$ e Carlos Cardoso Machado ${ }^{3}$
}

\begin{abstract}
RESUMO - Este trabalho buscou ilustrar um cenário de alternativas na cobrança de impostos, baseado em diferentes métodos de depreciação. A referência foi a atividade de transporte florestal, sendo o estudo desenvolvido para o caminhão bitrem. O fluxo de caixa foi calculado antes e depois dos impostos, sendo estes determinados com base num porcentual incidido sobre a renda tributável, que variou em função das metodologias utilizadas no cálculo da depreciação. Após a elaboração do fluxo de caixa pós-impostos, realizou-se um estudo econômico utilizando o Valor Presente Líquido (VPL). O impacto do imposto de renda ocasionou retrações na VPL de $53,36 \%, 52,22 \%, 50,54 \%, 48,07 \%$ e $47,72 \%$ quando se consideraram, respectivamente, os seguintes métodos de depreciação: soma inversa dos dígitos, fundo de renovação, linear, exponencial e soma dos dígitos. A metodologia mais compatível para o bitrem foi a mesma que apresentou maior VPL (soma dos dígitos), indicando que o método adotado pelo governo pode acarretar sobrecarga tributária. Isso reflete a necessidade de se estabelecerem critérios mais justos na cobrança de impostos, através da adoção de metodologias mais adequadas às realidades dos equipamentos.
\end{abstract}

Palavras-chave: Impostos, métodos de depreciação e avaliação econômica.

\section{INFLUENCE OF METHODS FOR CALCULATING DEPRECIATION ON THE INCOME TAX AND CASH FLOW OF A FOREST TRANSPORTION ACTIVITY}

\begin{abstract}
This work sought to illustrate a scenario of alternatives to taxation, based on different methods of depreciation. The parameter was the forest transportation activity, being the study developed for the Bitrem truck. Cash flow was calculated before and after taxation, which values were determined on the basis of a percentage on the taxable income, varying according to the methodologies used for calculating depreciation. After drawing up the post-taxation cash flow, an economic study was carried out using Net Present Value $(N P V)$. The impact of the income tax caused a reduction in NPV of 53.36\%, 52.22\%, 50.54\%, 48.07\% and $47.72 \%$ respectively when considering the following methods of depreciation: inverse sum of the digits; sinking fund; linear; exponential and sum of the digits. The most suitable methodology for the forest truck was the same that gave the largest NPV (sum of the digits), indicating that the method adopted by the government can result in tax overload. This shows the need for establishing better taxation rules, using more suitable methods for these types of machines.
\end{abstract}

Keywords: Taxes, depreciation methods and economic evaluation.

\footnotetext{
${ }^{1}$ Recebido em 29.05.2006 e aceito para publicação em 22.01.2007.

${ }^{2}$ Programa de Pós-Graduação em Ciência Florestal da Universidade Federal de Viçosa. E-mail: <luiscarlos_ufv@yahoo.com.br>.

${ }^{3}$ Departamento de Engenharia Florestal da Universidade Federal de Viçosa. E-mail: <marlosil@ ufv.br>.
} 


\section{INTRODUÇÃO}

A variedade de metodologias para o cálculo da depreciação constitui num aspecto imprescindível para o estabelecimento de critérios específicos e mais compatíveis no cálculo do imposto de renda. Os métodos de depreciação influenciam a decisão de investimento, uma vez que afetam a distribuição do imposto de renda a ser pago ao longo da vida útil dos bens (REZENDE e VALVERDE, 1997). A depreciação é a diminuição do valor de um bem, resultante do desgaste pelo uso, pela ação da natureza ou pela obsolescência normal, correspondente à perda do valor dos equipamentos com o passar do tempo e o uso (HIRSCHFELD, 1992). Noronha (1981) definiu esse termo como uma reserva contábil destinada a prover fundos necessários para a substituição do capital investido em bens produtivos de longa duração, em função de desgaste e, ou, obsolescência. É uma forma que a empresa usa para recuperar o bem de capital repondo-o quando este se torna economicamente inútil. Padoveze (1991) definiu a depreciação financeira como o valor ou custo da recuperação dos valores investidos no negócio. Esse custo, ou depreciação, deve ser considerado na análise do retorno do investimento. Existem vários métodos para esse cálculo, o que se justifica, uma vez que os equipamentos estão sujeitos a diferentes tipos de depreciação (física, funcional, acidental). No caso deste trabalho, em que se estudou um veículo de transporte florestal, a depreciação parece estar mais relacionada com o aspecto funcional. Contudo, a escolha de um ou outro método muitas vezes não é tão simples como pode parecer. $\mathrm{O}$ perfeito entendimento das utilidades de um ativo, sua valorização e o delineamento da perda do potencial de uso são condições básicas para determinar qual dos métodos deverá ser utilizado. Hendriksen e van Breda (1992) relataram sobre a impossibilidade de defender um método de alocação como superior ao outro, tendo em vista que não há base teórica para se preferir um em detrimento de outro. De acordo com esses mesmos autores, antes de definir um método é preciso efetuar as seguintes estimativas: a valorização (custo ou outra base) do ativo quando adquirido ou uma reavaliação dele em uma data subseqüente; a expectativa de vida desse ativo; e o valor residual, ou valor de liquidação final da vida útil desse ativo.

O método de depreciação exerce influência direta sobre a renda tributável, podendo, dessa forma, influenciar significativamente o fluxo de caixa e, conseqüentemente, a viabilidade do projeto em questão. Para equipamentos que apresentam depreciação acelerada, um dos métodos mais compatíveis para o cálculo do imposto de renda seria o exponencial. Nesses casos, o emprego do método linear (normalmente adotado pelo governo) poderá acarretar excessos de tributos. A depreciação acelerada ocorre nos casos em que, nos primeiros anos após o investimento, a depreciação é maior do que no método linear. Entretanto, se a depreciação não se caracterizar como acelerada, poderá, nessas condições, ocorrer uma subestimação da carga tributária, com um respectivo aumento do lucro por parte da empresa. O direcionamento desse enfoque econômico para operação de transporte florestal é de grande importância, dada a grande representatividade dessa atividade no custo final da madeira (MACHADO e LOPES, 2000).

Portanto, o objetivo deste estudo foi analisar o fluxo de caixa para a atividade de transporte florestal, através do Valor Presente Líquido (VPL), tomandose como base metodologias distintas no cálculo da depreciação.

\section{MATERIAL E MÉTODOS}

\subsection{Aspectos relacionados ao equipamento}

O Bitrem florestal normalmente é empregado para o transporte longitudinal de toras oriundas de reflorestamentos (PISSETTI, 2001). Sua capacidade de carga é de 36 toneladas, trafegando tanto em estradas principais quanto naquelas vicinais (áreas de domínio das empresas). Nesse último caso, é necessário que o local, ou melhor, o traçado das estradas, atenda a alguns quesitos para que se possa trafegar sem maiores problemas, como declividade favorável, condições de visibilidade e largura satisfatórias e, sobretudo, boas condições de estabilidade.

\section{2. Considerações gerais}

Foi analisado neste trabalho o fluxo de caixa para a atividade de transporte florestal, dando ênfase aos custos de impostos incididos em função de cinco metodologias de cálculo de depreciação. O trabalho foi realizado mediante um estudo de caso, tomandose como parâmetro um caminhão Volvo NH 12 e dois semi-reboques, sendo o conjunto denominado Bitrem. Os dados utilizados para o cálculo das receitas e dos custos do referido veículo, ou seja, aqueles necessários 
para elaboração do fluxo de caixa, foram extraídos de um trabalho científico realizado por Freitas et al. (2004), os quais estudaram detalhadamente esse tipo de composição florestal.

As receitas anuais foram obtidas através da multiplicação da quilometragem anual $(120.000 \mathrm{~km})$ pelo preço do quilômetro rodado $(\mathrm{R} \$ 1,90)$. Os custos anuais foram obtidos através da multiplicação do custo por hora efetiva $(\mathrm{R} \$ 76,05)$ e pelo número de horas trabalhadas no ano (2.160 horas). No trabalho desenvolvido por Freitas et al. (2004), o custo operacional foi calculado utilizando-se três metodologias, sendo que uma delas (Battistella/Scânia) serviu de parâmetro para o referido estudo. Com base nesse método, encontrou-se um valor de US $\$ 33,80 /$ ha, que pela transformação monetária (1US\$ $=\mathrm{R} \$ 2,25)$, resultou em $\mathrm{R} \$ 76,05 / \mathrm{ha}$. O número de horas efetivas de trabalho por ano foi determinado através da divisão da quilometragem anual $(120.000 \mathrm{~km})$ pela velocidade média do caminhão $(55,55 \mathrm{~km} / \mathrm{hora})$, chegandose a um valor aproximado de $2.160 \mathrm{ha} / \mathrm{ano}$.

Com base nos dados anuais de receitas (120.000 $* 1,90)$ e custos $(76,05 * 2.160)$, calculou-se o fluxo de caixa (antes dos impostos). A partir daí, foram calculadas as despesas com tributos, representando $30 \%$ da renda tributável e variando em função do método utilizado para o cálculo da depreciação (Tabelas 1, 2, $3,4$ e 5$)$.

Foram contemplados os seguintes métodos de depreciação: linear, soma dos dígitos (cole), soma inversa dos dígitos e fundo de renovação e exponencial. Os métodos, bem como os dados utilizados no cálculo da depreciação do Bitrem, foram os relatados nos tópicos subseqüentes.

\subsubsection{Métodos de Cálculo de Depreciação}

\subsubsection{Linear}

Consiste num método em que a taxa anual de depreciação é calculada, dividindo-se o custo inicial menos o valor final, pelo número de anos de duração provável (OLIVEIRA, 2000).

$D=\frac{V_{o}-V_{r}}{n} \Rightarrow D=\frac{200.000-40.000}{10} \Rightarrow D=16.000 \Rightarrow D_{1}=D_{2} \ldots . . D_{10}=16.000$ em que:

$\mathrm{D}=$ depreciação; $\mathrm{Va}=$ valor de aquisição; $\mathrm{Vr}=$ valor residual; e $\mathrm{N}=$ vida útil *(em anos).
* A vida útil pode ser o período durante o qual se espera que um ativo depreciável seja utilizado pela empresa ou o número de unidades de produção ou outras unidades similares que a empresa espera obter desse ativo (PEREIRA, 1999).

\subsubsection{Exponencial}

O método exponencial é coerente com o argumento de que a contribuição de dado bem para geração de renda é maior nos anos iniciais de sua vida útil e decresce com o uso (REZENDE e VALVERDE, 1997).

$$
\begin{aligned}
& V_{n}=V_{0}(1-T)^{n} \quad T=1-\sqrt[N]{\frac{V_{n}}{V_{0}}} \Rightarrow T=1-\sqrt[10]{\frac{40.000}{200.000}} \Rightarrow T=14,87 \\
& V_{0}=200.000(1-0,1487)^{0} \\
& V_{1}=200.000(1-0,1487)^{1} \because D_{1}=V_{0}-V_{1} \Rightarrow D_{1}=29.740,00 \\
& V_{2}=200.000(1-0,1487)^{2} \because D_{2}=V_{1}-V_{2} \Rightarrow D_{2}=25.317,66 \\
& \ldots \\
& V_{9}=200.000(1-0,1487)^{9} \\
& V_{10}=200.000(1-0,1487)^{10} \because D_{10}=V_{9}-V_{10} \Rightarrow D_{10}=6.983,09
\end{aligned}
$$

em que,

$\mathrm{V}_{\mathrm{n}}=$ valor do veículo no ano $\mathrm{n} ; \mathrm{V}_{0}=$ valor inicial do caminhão; $T$ = taxa porcentual anual de depreciação; $\mathrm{n}=$ ano em consideração; $\mathrm{e} \mathrm{N}=$ vida útil estimada do veículo.

\subsubsection{Soma dos dígitos (cole)}

É um método que propicia carga anual de depreciação decrescente, aplicada sempre ao mesmo valor obtido da diferença entre o valor de aquisição e o valor de descarte como sucata (COSENTINO, 2004), ou seja:

$$
\begin{aligned}
& D=\frac{N}{S D}\left(V_{0}-V_{n}\right) \quad S D=1+2+3+4+5+6+7+8+9+10 \Rightarrow S D=55 \\
& D_{1}=\frac{10}{55}(200.000-40.000) \Rightarrow D_{1}=29.090,91 \\
& D_{2}=\frac{9}{55}(200.000-40.000) \Rightarrow D_{2}=26.181,82 \\
& \ldots \\
& D_{10}=\frac{1}{55}(200.000-40.000) \Rightarrow D_{10}=2.909,09
\end{aligned}
$$

em que:

$\mathrm{D}=$ depreciação $; \mathrm{SD}=$ soma dos dígitos $; \mathrm{e} \mathrm{N}=$ ano de vida útil, em consideração.

R. Árvore, Viçosa-MG, v.31, n.2, p.257-264, 2007 


\subsubsection{Soma inversa dos dígitos}

É um método que, ao contrário da soma dos dígitos, propicia uma carga anual de depreciação crescente (REZENDE e VALVERDE, 1997), ou seja:

$D=\frac{N}{S D}\left(V_{0}-V_{n}\right)$

$D_{1}=\frac{1}{55}(200.000-40.000) \Rightarrow D_{1}=2.909,09$

$D_{2}=\frac{2}{55}(200.000-40.000) \Rightarrow D_{2}=5.818,18$

$D_{10}=\frac{10}{55}(200.000-40.000) \Rightarrow D_{10}=29.090,91$

em que:

$\mathrm{D}=$ depreciação; $\mathrm{SD}=$ soma dos dígitos; e $\mathrm{N}=$ ano de vida útil em consideração.

\subsubsection{Fundo de renovação (sinking fund)}

Método em que o cálculo da depreciação é feito de forma a compor uma reserva de capital, sobre o qual incidem juros, de forma que ao final da vida econômica da máquina o recurso seja suficiente para a sua reposição (COSENTINO, 2004; IUDÍCIBUS, 2000 ), isto é:

$$
\begin{aligned}
& V_{n}=\frac{R\left[(1+i)^{n}-1\right]}{i} \Rightarrow R=\frac{\left(V_{0}-V_{r}\right)}{\frac{\left[(1+i)^{n}-1\right]}{i}} \\
& R=\frac{(200.000-40.000)}{\frac{\left[(1+0,12)^{10}-1\right]}{0,12}} \Rightarrow R=9.117,47 \\
& D_{n}=R(1+i)^{n} \\
& D_{1}=9.117,47(1+0,12)^{0} \Rightarrow D_{1}=9.117,47 \\
& D_{2}=9.117,47(1+0,12)^{1} \Rightarrow D_{2}=10.211,56 \\
& \ldots \\
& D_{10}=9.117,47(1+0,12)^{9} \Rightarrow D_{10}=25.283,46
\end{aligned}
$$

em que:

$\mathrm{D}_{\mathrm{n}}=$ depreciação no ano $\mathrm{n} ; \mathrm{R}=$ parcela de depreciação anual; $\mathrm{n}=$ vida útil em anos; e $\mathrm{i}=$ taxa anual de juros (12\% a.a.).

\subsection{Análise econômica}

O método de avaliação econômica adotado no estudo foi o Valor Presente Líquido (VPL). Esta análise foi realizada tomando-se como base o fluxo de caixa pósimpostos (Tabelas 1, 2, 3, 4 e 5). Utilizou-se, portanto, a seguinte fórmula para análise do projeto:

$$
\mathrm{VPL}=\sum_{T=0}^{n} R_{T} e^{-r T}-\sum_{T=0}^{n} C_{T} e^{-r T}
$$

em que:

$$
\begin{aligned}
& \text { VPL: valor presente líquido; } \\
& \text { C: custo no período T; } \\
& \text { R: receita no período; } \\
& \text { T: período em que ocorrem R e C; } \\
& \text { r: taxa real de desconto; e } \\
& \text { e: base do logaritmo. }
\end{aligned}
$$

\section{RESULTADOS E DISCUSSÃO}

O fato de não se considerar o Imposto de Renda (IR) fez que o VPL atingisse um valor de $\mathrm{R} \$ 160.100,01$. Considerando, portanto, o I.R., esse valor retraiu para $53,36 \% ; 52,22 \% ; 50,54 \% ; 48,07 \%$; e $47,72 \%$, respectivamente, quando se consideraram os seguinte métodos de depreciação: Soma Inversa dos Dígitos; Fundo de Renovação; Linear; Exponencial e Soma dos Dígitos (Figura 1). Embora tenha havido diferenciação, a depreciação total no final da vida útil do ativo apresentou valores bastante próximos para os métodos analisados. O método de depreciação Linear, adotado pelo governo para o cálculo do imposto de renda, foi o terceiro mais representativo, em termos de tributos, para a atividade de transporte florestal (Figura 1). Esse método é o de mais larga utilização pelos contadores, devido à sua simplicidade de cálculo, além de ser aceito, também, pela legislação fiscal (IUDIBUS, 2000). A taxação mais expressiva foi observada quando se utilizou o método denominado Soma Inversa dos Dígitos (Figura 1 e Tabela 4). A depreciação calculada pelos métodos Exponencial e Soma dos Dígitos (Cole) proporcionou uma acentuada desvalorização do caminhão florestal nos primeiros anos, o que contribuiu para a redução da renda tributável e, automaticamente, da taxa de impostos. Esse aspecto favoreceu o fluxo de caixa, explicando os maiores valores do VPL para tais metodologias (Figura 1). Embora os outros métodos de depreciação para efeito do cálculo do imposto de renda não sejam comumente utilizados pelo governo, o trabalho mostrou que a utilização de outras metodologias poderia influenciar a taxação dos impostos. 


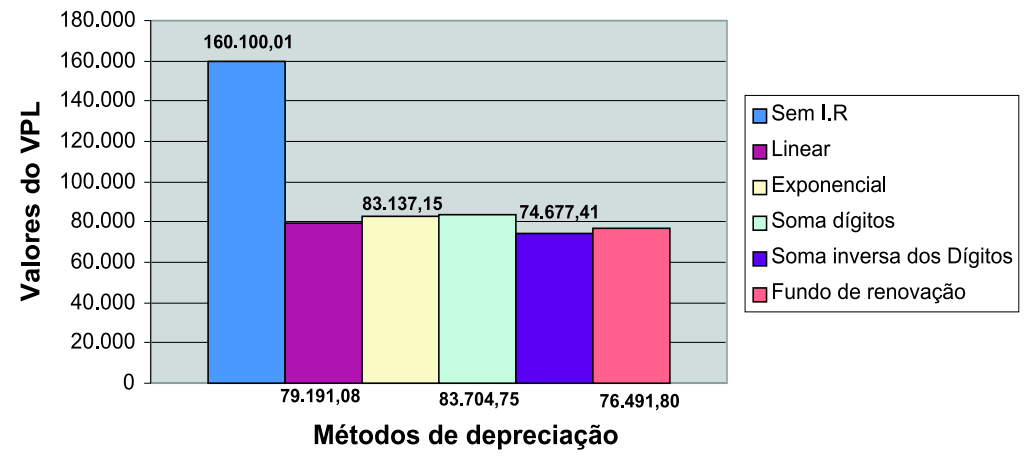

Figura 1 - Análise do VPL em relação ao fluxo de caixa pós-impostos, na atividade de transporte florestal, considerandose metodologias distintas no cálculo da depreciação.

Figure 1 - NPV analysis regarding post-taxation cash flow, for a forest transportation activity, considering different methods for calculating depreciation.

O método Cole (Soma dos Dígitos) tem mostrado, em alguns trabalhos, maior proximidade do valor de revenda para veículos de transporte florestal (REZENDE e VALVERDE, 1997). Coincidentemente, esse método apresentou maior valor de VPL, indicando que, neste estudo, pode estar havendo uma cobrança excessiva de tributos por parte do governo. Em outras palavras, se o cálculo do imposto de renda fosse feito com base na metodologia adequada, a taxa de encargos seria menor. Isso pode ser demonstrado através da Figura 1, quando são comparados os seguintes valores do VPL: R $\$ 83.137,15$ (soma dos dígitos) e $\mathrm{R} \$ 79.191,08$ (linear).

Além dos métodos para o cálculo da depreciação, outro fator que pode afetar a viabilidade do projeto é a taxa de juros. Essa variável assumiu grande importância neste estudo, principalmente em virtude do critério de análise econômica adotado (VPL). Os resultados deste trabalho foram obtidos com base numa taxa anual de $12 \%$.

Considerou-se neste estudo um rendimento constante do veículo florestal durante sua vida útil. Porém, em situações reais isso poderia ocorrer apenas nos primeiros anos de uso, uma vez que, com o passar do tempo, a tendência é de haver uma redução na eficiência operacional do equipamento.

A aplicação de diferentes métodos de depreciação resultou numa diferenciação do comportamento do fluxo de caixa. Nesse aspecto, observaram-se tendências constante (depreciação linear), decrescente (exponencial e soma dos dígitos) e crescente (fundo de renovação e soma inversa dos dígitos) (Figura 2). Esses comportamentos apresentados pelos fluxos de caixa mostraram, da mesma forma, as tendências dos respectivos métodos de depreciação. Tais tendências foram, inclusive, o mecanismo abordado por Iudibus (2000) na classificação desses métodos, tendo, portanto, evidenciado os seguintes grupos: método de cotas constantes (Quadro 1), método de cotas variáveis, método de cotas crescentes (Quadros 4 e 5) e método de cotas decrescentes (Quadros 2 e 3).

A aplicação do método das cotas constantes (linear) traz vantagens no início, pelo fato de os produtos receberem parcela muito pequena da depreciação, e uma desvantagem no final da vida útil, já que nesse período a depreciação teria representatividade maior nos custos do produto. Contudo, nesta avaliação esse método acabou apresentando viabilidade intermediária quando aplicado no cálculo da depreciação (Figura 1). Os métodos de comportamento crescente, como soma inversa dos dígitos e fundo de renovação (Figura 2), quando utilizados para o cálculo do imposto de renda, não proporcionaram bons resultados (Figura 1). Oleiro (1999) relatou, no entanto, a importância da utilização dos métodos de depreciação de cotas decrescentes para o cálculo do imposto de renda. Segundo esse mesmo autor, a utilização desses métodos apresentaria vantagens fiscais pela redução do lucro tributável. Esse relato está de acordo com os resultados deste trabalho (Figura 1), em que foi observado maior valor de VPL para os respectivos métodos (exponencial e soma dos dígitos).

R. Árvore, Viçosa-MG, v.31, n.2, p.257-264, 2007 


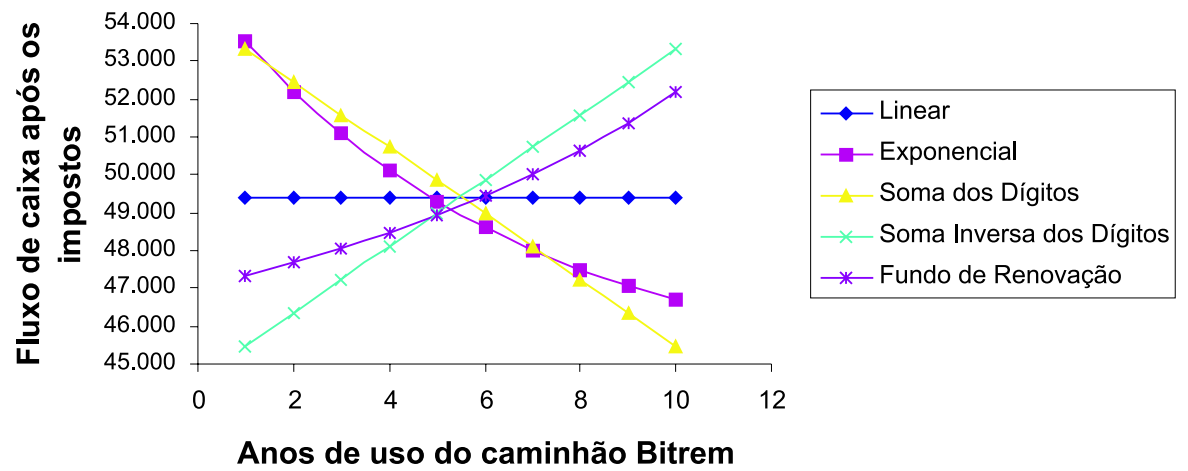

Figura 2 - Comportamento do fluxo de caixa pós-impostos na atividade de transporte florestal, tomando-se como referência cinco metodologias para o cálculo da depreciação.

Figure 2-Behavior of post-taxation cash flow, for a forest transportation activity, considering different methods for calculating depreciation.

Quadro 1 - Influência do método de depreciação "linear" no imposto de renda e no fluxo de caixa de uma atividade de transporte florestal, considerando-se o caminhão Bitrem

Table 1 - Influence of the linear depreciation method on income tax and cash flow of a forest transportation activity, for bitrem truck

\begin{tabular}{|c|c|c|c|c|c|}
\hline Ano & $\begin{array}{c}\text { Fluxo de caixa antes } \\
\text { dos impostos } \\
(\mathrm{R} \$)(\mathbf{a})\end{array}$ & $\begin{array}{c}\text { Despesas com } \\
\text { depreciação } \\
(\mathrm{R} \$)(\mathbf{b})\end{array}$ & $\begin{array}{c}\text { Renda Tributável } \\
(\mathrm{RT})(\mathrm{R} \$) \\
(\mathbf{a}-\mathbf{b})\end{array}$ & $\begin{array}{c}\text { Imposto de renda } \\
(30 \% \text { de } \mathrm{RT}) \\
(\mathrm{R} \$)(\mathbf{c})\end{array}$ & $\begin{array}{c}\text { Fluxo de caixa } \\
\text { após os impostos } \\
(\mathrm{R} \$)(\mathbf{a - c})\end{array}$ \\
\hline 0 & $-200.000,00$ & & & & $-200.000,00$ \\
\hline 1 & $63.732,00$ & $16.000,00$ & $47.732,00$ & $14.319,60$ & $49.412,40$ \\
\hline 2 & $63.732,00$ & $16.000,00$ & $47.732,00$ & $14.319,60$ & $49.412,40$ \\
\hline 3 & $63.732,00$ & $16.000,00$ & $47.732,00$ & $14.319,60$ & $49.412,40$ \\
\hline 4 & $63.732,00$ & $16.000,00$ & $47.732,00$ & $14.319,60$ & $49.412,40$ \\
\hline 5 & $63.732,00$ & $16.000,00$ & $47.732,00$ & $14.319,60$ & $49.412,40$ \\
\hline 6 & $63.732,00$ & $16.000,00$ & $47.732,00$ & $14.319,60$ & $49.412,40$ \\
\hline 7 & $63.732,00$ & $16.000,00$ & $47.732,00$ & $14.319,60$ & $49.412,40$ \\
\hline 8 & $63.732,00$ & $16.000,00$ & $47.732,00$ & $14.319,60$ & $49.412,40$ \\
\hline 9 & $63.732,00$ & $16.000,00$ & $47.732,00$ & $14.319,60$ & $49.412,40$ \\
\hline 10 & $63.732,00$ & $16.000,00$ & $47.732,00$ & $14.319,60$ & $49.412,40$ \\
\hline
\end{tabular}

Quadro 2 - Influência do método de depreciação "exponencial" no imposto de renda e no fluxo de caixa de uma atividade de transporte florestal, considerando-se o caminhão bitrem

Table 2 - Influence of the exponential depreciation method on income tax and cash flow of a forest transportation activity, for bitrem truck

\begin{tabular}{|c|c|c|c|c|c|}
\hline$\overline{\text { Ano }}$ & $\begin{array}{c}\text { Fluxo de caixa antes } \\
\text { dos impostos } \\
(\mathrm{R} \$)(\mathbf{a})\end{array}$ & $\begin{array}{c}\text { Despesas com } \\
\text { depreciação } \\
(\mathrm{R} \$)(\mathbf{b})\end{array}$ & $\begin{array}{c}\text { Renda Tributável } \\
(\mathrm{RT})(\mathrm{R} \$) \\
(\mathbf{a}-\mathbf{b})\end{array}$ & $\begin{array}{c}\text { Imposto de renda } \\
(30 \% \text { de } \mathrm{RT}) \\
(\mathrm{R} \$)(\mathbf{c})\end{array}$ & $\begin{array}{c}\text { Fluxo de caixa } \\
\text { após os impostos } \\
(\mathrm{R} \$)(\mathbf{a}-\mathbf{c})\end{array}$ \\
\hline 0 & $-200.000,00$ & & & & $-200.000,00$ \\
\hline 1 & $63.732,00$ & $29.740,00$ & $33.992,00$ & $10.197,60$ & $53.534,40$ \\
\hline 2 & $63.732,00$ & $25.317,66$ & $38.414,34$ & $11.524,30$ & $52.207,70$ \\
\hline 3 & $63.732,00$ & $21.552,93$ & $42.179,07$ & $12.653,72$ & $51.078,28$ \\
\hline 4 & $63.732,00$ & $18.348,01$ & $45.383,99$ & $13.615,20$ & $50.116,80$ \\
\hline 5 & $63.732,00$ & $15.619,66$ & $48.112,34$ & $14.433,70$ & $49.298,30$ \\
\hline 6 & $63.732,00$ & $13.297,01$ & $50.434,99$ & $15.130,50$ & $48.601,50$ \\
\hline 7 & $63.732,00$ & $11.319,75$ & $52.412,25$ & $15.723,68$ & $48.008,32$ \\
\hline 8 & $63.732,00$ & $9.636,50$ & $54.095,5$ & $16.228,65$ & $47.503,35$ \\
\hline 9 & $63.732,00$ & $8.203,55$ & $55.528,45$ & $16.658,54$ & $47.073,46$ \\
\hline 10 & $63.732,00$ & $6.983,09$ & $56.748,31$ & $17.024,49$ & $46.707,51$ \\
\hline
\end{tabular}

R. Árvore, Viçosa-MG, v.31, n.2, p.257-264, 2007 
Quadro 3 - Influência do método de depreciação "soma dos dígitos" no imposto de renda e no fluxo de caixa de uma atividade de transporte florestal, considerando-se o caminhão bitrem

Table 3 - Influence of the sum of the digits depreciation method on income tax and cash flow of a forest transportation activity, for bitrem truck

\begin{tabular}{|c|c|c|c|c|c|}
\hline$\overline{A n o}$ & $\begin{array}{c}\text { Fluxo de caixa antes } \\
\text { dos impostos } \\
(\mathrm{R} \$)(\mathbf{a})\end{array}$ & $\begin{array}{c}\text { Despesas com } \\
\text { depreciação } \\
(\mathrm{R} \$)(\mathbf{b})\end{array}$ & $\begin{array}{c}\text { Renda Tributável } \\
(\mathrm{RT})(\mathrm{R} \$) \\
(\mathbf{a}-\mathbf{b})\end{array}$ & $\begin{array}{c}\text { Imposto de renda } \\
(30 \% \text { de } \mathrm{RT}) \\
(\mathrm{R} \$)(\mathbf{c})\end{array}$ & $\begin{array}{c}\text { Fluxo de caixa } \\
\text { após os impostos } \\
(\mathrm{R} \$)(\mathbf{a}-\mathbf{c})\end{array}$ \\
\hline 0 & $-200.000,00$ & & & & $-200.000,00$ \\
\hline 1 & $63.732,00$ & $29.090,91$ & $34.641,09$ & $10.392,33$ & $53.339,67$ \\
\hline 2 & $63.732,00$ & $26.181,82$ & $37.550,18$ & $11.265,05$ & $52.466,95$ \\
\hline 3 & $63.732,00$ & $23.272,73$ & $40.459,27$ & $12.137,78$ & $51.594,22$ \\
\hline 4 & $63.732,00$ & $20.363,64$ & $43.368,36$ & $13.010,51$ & $50.721,49$ \\
\hline 5 & $63.732,00$ & $17.454,55$ & $46.277,45$ & $13.883,24$ & $49.848,76$ \\
\hline 6 & $63.732,00$ & $14.545,45$ & $49.186,55$ & $14.755,97$ & $48.976,03$ \\
\hline 7 & $63.732,00$ & $11.636,36$ & $52.095,64$ & $15.628,69$ & $48.103,31$ \\
\hline 8 & $63.732,00$ & $8.727,27$ & $55.004,73$ & $16.501,42$ & $47.230,58$ \\
\hline 9 & $63.732,00$ & $5.818,18$ & $57.913,82$ & $17.374,15$ & $46.357,85$ \\
\hline 10 & $63.732,00$ & $2.909,09$ & $60.822,91$ & $18.246,87$ & $45.485,13$ \\
\hline
\end{tabular}

Quadro 4 - Influência do método de depreciação "soma inversa dos dígitos" no imposto de renda e no fluxo de caixa de uma atividade de transporte florestal, considerando-se o caminhão bitrem

Table 4 - Influence of the inverse sum of the digits depreciation method on income tax and cash flow of a forest transportation activity, for bitrem truck

\begin{tabular}{cccccc}
\hline Ano & $\begin{array}{c}\text { Fluxo de caixa antes } \\
\text { dos impostos } \\
(\mathrm{R} \$)(\mathbf{a})\end{array}$ & $\begin{array}{c}\text { Despesas com } \\
\text { depreciação } \\
(\mathrm{R} \$)(\mathbf{b})\end{array}$ & $\begin{array}{c}\text { Renda Tributável } \\
(\mathrm{RT})(\mathrm{R} \$) \\
(\mathbf{a}-\mathbf{b})\end{array}$ & $\begin{array}{c}\text { Imposto de renda } \\
(30 \% \text { de RT) } \\
(\mathrm{R} \$)(\mathbf{c})\end{array}$ & $\begin{array}{c}\text { Fluxo de caixa } \\
\text { após os impostos } \\
(\mathrm{R} \$)(\mathbf{a - c})\end{array}$ \\
\hline 0 & $-200.000,00$ & & & & $-200.000,00$ \\
1 & $63.732,00$ & $2.909,09$ & $60.822,91$ & $18.246,87$ & $45.485,13$ \\
2 & $63.732,00$ & $5.818,18$ & $57.913,82$ & $17.374,15$ & $46.357,85$ \\
3 & $63.732,00$ & $8.727,27$ & $55.004,73$ & $16.501,42$ & $47.230,58$ \\
4 & $63.732,00$ & $11.636,36$ & $52.095,64$ & $15.628,69$ & $48.103,31$ \\
5 & $63.732,00$ & $14.545,45$ & $49.186,55$ & $14.755,96$ & $48.976,04$ \\
6 & $63.732,00$ & $17.454,54$ & $46.277,45$ & $13.883,24$ & $49.848,76$ \\
7 & $63.732,00$ & $20.363,64$ & $43.368,36$ & $13.010,51$ & $50.721,49$ \\
8 & $63.732,00$ & $23.272,73$ & $40.459,27$ & $12.137,78$ & $51.594,22$ \\
9 & $63.732,00$ & $26.181,82$ & $37.550,18$ & $11.265,05$ & $52.466,95$ \\
10 & $63.732,00$ & $29.090,91$ & $34.641,09$ & $10.392,33$ & $53.339,67$ \\
\hline
\end{tabular}

Quadro 5 - Influência do método de depreciação "fundo de renovação" no imposto de renda e no fluxo de caixa de uma atividade de transporte florestal, considerando-se o caminhão bitrem

Table 5 - Influence of the sinking fund depreciation method on income tax and cash flow of a forest transportation activity, for bitrem truck

\begin{tabular}{ccccc}
\hline Ano & $\begin{array}{c}\text { Fluxo de caixa antes } \\
\text { dos impostos } \\
(\mathrm{R} \$)(\mathbf{a})\end{array}$ & $\begin{array}{c}\text { Despesas com } \\
\text { depreciação } \\
(\mathrm{R} \$)(\mathbf{b})\end{array}$ & $\begin{array}{c}\text { Renda Tributável } \\
(\mathrm{RT})(\mathrm{R} \$) \\
(\mathbf{a}-\mathbf{b})\end{array}$ & $\begin{array}{c}\text { Imposto de renda } \\
(30 \% \text { de RT) } \\
(\mathrm{R} \$)(\mathbf{c})\end{array}$ \\
\hline 0 & $-200.000,00$ & & & $\begin{array}{c}\text { Fluxo de caixa } \\
\text { após os impostos } \\
(\mathrm{R} \$)(\mathbf{a}-\mathbf{c})\end{array}$ \\
1 & $63.732,00$ & $9.117,47$ & $54.614,53$ & $-200.000,00$ \\
2 & $63.732,00$ & $10.211,56$ & $53.520,44$ & $16.384,36$ \\
3 & $63.732,00$ & $11.436,95$ & $52.295,05$ & $16.056,13$ \\
4 & $63.732,00$ & $12.809,39$ & $50.922,61$ & $15.688,52$ \\
5 & $63.732,00$ & $14.346,51$ & $49.385,49$ & $15.276,78$ \\
6 & $63.732,00$ & $16.068,09$ & $47.663,91$ & $14.815,65$ \\
7 & $63.732,00$ & $17.996,27$ & $45.735,73$ & $14.299,17$ \\
8 & $63.732,00$ & $20.155,82$ & $43.576,18$ & $13.720,72$ \\
9 & $63.732,00$ & $22.574,52$ & $41.157,48$ & $13.072,85$ \\
10 & $63.732,00$ & $25.283,46$ & $38.448,54$ & $12.347,24$ \\
\hline
\end{tabular}




\section{CONCLUSÕES}

Com base na presente avaliação, chegou-se às seguintes conclusões:

- Os melhores resultados, economicamente, foram obtidos quando se aplicaram os métodos de depreciação de cotas decrescentes (exponencial e soma dos dígitos).

-A depreciação total do veículo florestal, computado pelo somatório das cotas de depreciação, apresentou valores semelhantes nos cinco métodos analisados.

- O método de depreciação adotado pelo governo proporcionou, para o equipamento em estudo, uma carga de impostos superior em relação ao método mais adequado (soma dos dígitos).

- Concluiu-se que é possível estabelecer uma carga tributária mais justa, através da utilização de metodologias diferenciadas de depreciação no cálculo do imposto de renda.

- A taxa de juros mostrou-se como uma variável de grande importância no cálculo da depreciação, principalmente em virtude do critério adotado para a análise econômica (VPL) e do longo período de duração do projeto (vida útil do veículo florestal).

\section{REFERÊNCIAS}

Cosentino, R. M. A. Modelo empírico de depreciação para tratores agrícolas de rodas. 2004. 80f. Dissertação (Mestrado em Agronomia) - Escola Superior de Agricultura "Luiz de Queiroz”, Piracicaba, 2004.

FREITAS, L. C. et al. Estudo comparativo envolvendo três métodos de calculo de custo operacional do caminhão Bitrem. Revista Árvore. v.28, n.6, p.855-863, 2004.

HENDRIKSEN, E. S.; van BREDA, M. F. Accouting theory. Illinois: Richard D. Irwin, 1992. 734p.

IUdíCIBUS, S. Teoria da contabilidade. 6 . ed. São Paulo: Atlas, 2000. 350p.

R. Árvore, Viçosa-MG, v.31, n.2, p.257-264, 2007
HIRSCHFELD, H. Engenharia econômica e análise de custos. 5. ed. São Paulo: Atlas, 1992. 465p.

MACHADO, C. C.; LOPES, E. S. Análise da influência do comprimento de toras de eucalipto na produtividade e custo da colheita e transporte florestal. Revista Cerne, v.6, n.2, p.124-129, 2000.

NORONHA, J.F. Projetos agropecuários: administração financeira, orçamento e viabilidade econômica. São Paulo: Atlas, 1981. 274p.

OLEIRO, W. N. Métodos de depreciação.

Revista do Conselho Regional de Contabilidade do Rio Grande do Sul. n.96, p.18-24,1999.

OLIVEIRA, M. D. M. Custo operacional e ponto de renovação de tratores agrícolas de pneus: avaliação de uma frota. 2000. 150f. Dissertação (Mestrado em Agronomia) - Escola Superior de Agricultura "Luiz de Queiroz”, Piracicaba, 2000.

PADOVEZE, C.L. Manual de contabilidade básica. 2. ed. São Paulo: Atlas, 1991. 294p.

PEREIRA, M.A.T.A. Recuperação dos custos pela depreciação. Anais de Contabilidade, Edição Especial da revista do NUSEG/UERJ, 1999.

PISSETTI, C.A. Tendências e perspectivas do uso do Bitrem no transporte de madeira no Brasil. In: SIMPÓSIO BRASILEIRO SOBRE COLHEITAE TRANSPORTE FLORESTAL, 5., 2001, Porto Seguro. Anais... Porto Seguro: SIF, 2001. p.152- 167.

REZENDE, J.L.P.; VALVERDE, S. R. Princípios de depreciação de máquinas e equipamentos. Revista árvore. v.21, n.1., p.99-111, 1997. 